University of Wollongong

Research Online

Faculty of Engineering and Information

Faculty of Engineering and Information

Sciences - Papers: Part B

Sciences

2019

\title{
A Monte Carlo study on the feasibility of real-time in vivo source tracking during ultrasound based HDR prostate brachytherapy treatments
}

Joel Poder

University of Wollongong, jp132@uowmail.edu.au

Dean L. Cutajar

University of Wollongong, deanc@uow.edu.au

Susanna Guatelli

University of Wollongong, susanna@uow.edu.au

Marco Petasecca

University of Wollongong, marcop@uow.edu.au

Andrew Howie

Royal North Shore Hospital

See next page for additional authors

Follow this and additional works at: https://ro.uow.edu.au/eispapers1

Part of the Engineering Commons, and the Science and Technology Studies Commons

Research Online is the open access institutional repository for the University of Wollongong. For further information contact the UOW Library: research-pubs@uow.edu.au 


\section{A Monte Carlo study on the feasibility of real-time in vivo source tracking during ultrasound based HDR prostate brachytherapy treatments}

\section{Abstract \\ Purpose}

This study aims to assess the accuracy of in-vivo source tracking during real-time trans-rectal ultrasound (TRUS) based high dose rate (HDR) prostate brachytherapy (pBT) through Monte Carlo simulations of multiple HDR pBT treatments with a two-dimensional (2D) diode array, the Magic Plate 900 (MP900), embedded below the patient in a carbon-fibre couch.

\section{Method}

Monte Carlo simulations of source positions representing three separate real-time TRUS based HDR pBT treatments were performed using the Geant4 toolkit. For each source position, an Ir-192 source was simulated inside a voxelized patient geometry. Dose deposited from each source position to the MP900 diodes was used to perform source tracking, and the MP900 calculated position compared to known source positions from the treatment plan. Thresholding techniques were implemented to improve source tracking accuracy with the TRUS probe present.

\section{Results}

The average three-dimensional source position reconstruction discrepancy was $11.9 \pm 2.4 \mathrm{~mm}$ and $1.5 \pm$ $0.3 \mathrm{~mm}$ with and without the TRUS probe, respectively. Thresholding techniques improved the source position reconstruction discrepancy in the presence of the TRUS probe to $1.8 \pm 0.4 \mathrm{~mm}$.

\section{Conclusion}

Inclusion of the TRUS probe inside the patient negatively affects source tracking accuracy when using the MP900 diode array for HDR pBT verification. Modification of the source tracking algorithm using thresholding techniques improves source tracking in the presence of the TRUS probe, achieving similar accuracy as when the TRUS probe is not present. This study demonstrates that accurate in-vivo source tracking during real-time TRUS based HDR pBT is feasible using the Magic Plate system.

\section{Disciplines \\ Engineering | Science and Technology Studies}

\section{Publication Details}

Poder, J., Cutajar, D., Guatelli, S., Petasecca, M., Howie, A., Bucci, J., Carrara, M. \& Rosenfeld, A. (2019). A Monte Carlo study on the feasibility of real-time in vivo source tracking during ultrasound based HDR prostate brachytherapy treatments. Physica Medica: an international journal devoted to the applications of physics to medicine and biology, 59 30-36.

\section{Authors}

Joel Poder, Dean L. Cutajar, Susanna Guatelli, Marco Petasecca, Andrew Howie, Joseph A. Bucci, Mauro Carrara, and Anatoly B. Rosenfeld 


\section{ABSTRACT}

Purpose: This study aims to assess the accuracy of in-vivo source tracking during real-time trans-rectal ultrasound (TRUS) based high dose rate (HDR) prostate brachytherapy (pBT) through Monte Carlo simulations of multiple HDR pBT treatments with a two-dimensional (2D) diode array, the Magic Plate 900 (MP900), embedded below the patient in a carbon-fibre couch.

Method: Monte Carlo simulations of source positions representing three separate real-time TRUS based HDR pBT treatments were performed using the Geant4 toolkit. For each source position, an Ir192 source was simulated inside a voxelized patient geometry. Dose deposited from each source position to the MP900 diodes was used to perform source tracking, and the MP900 calculated position compared to known source positions from the treatment plan. Thresholding techniques were implemented to improve source tracking accuracy with the TRUS probe present.

Results: The average three-dimensional source position reconstruction discrepancy was $11.9 \pm 2.4 \mathrm{~mm}$ and $1.5 \pm 0.3 \mathrm{~mm}$ with and without the TRUS probe, respectively. Thresholding techniques improved the source position reconstruction discrepancy in the presence of the TRUS probe to $1.8 \pm 0.4 \mathrm{~mm}$.

Conclusion: Inclusion of the TRUS probe inside the patient negatively affects source tracking accuracy when using the MP900 diode array for HDR pBT verification. Modification of the source tracking algorithm using thresholding techniques improves source tracking in the presence of the TRUS probe, achieving similar accuracy as when the TRUS probe is not present. This study demonstrates that accurate in-vivo source tracking during real-time TRUS based HDR pBT is feasible using the Magic Plate system.

Key words: in-vivo, source tracking, real-time prostate brachytherapy, Magic Plate, MP900, Monte Carlo 


\section{I.INTRODUCTION}

High dose rate (HDR) prostate brachytherapy (pBT) treatments are widely practiced in the radiation oncology community, most commonly in combination with external beam radiotherapy (EBRT) in the form of a boost. There have been a number of recent studies showing improved quality of life outcome for combined EBRT and HDR brachytherapy compared with EBRT alone [1-3]. Furthermore, there have been several studies that reported favourable results using HDR brachytherapy as a monotherapy $[4,5]$.

HDR pBT treatment plans are traditionally performed with the use of a post-operative CT scan. The major drawback of this technique is that it necessitates movement of the patient off the operating table and out of the dorsal lithotomy position. Multiple studies have reported that movement of the patient in this manor may increase the risk of catheter displacement in the inferior direction, relative to the prostate, in the time between CT scanning and treatment [6, 7]. The use of transrectal ultrasound (TRUS) for treatment planning [8] has been shown to reduce the magnitude of these shifts, as well as improve visibility of the prostate and organs at risk (OARs) [9]. Modern brachytherapy treatment planning systems (BTPS) are able to use the ultrasound echoes of the implanted catheters for the definition of the catheter position within the patient, allowing for 'real-time' HDR pBT treatment planning [9]. Correct identification of catheter positions on these images however can be difficult, and there remains a need for comprehensive pre-treatment and/or in-vivo quality assurance (QA) [10-12].

Source tracking of dwell positions during HDR pBT treatments based on electronic portal imaging devices (EPIDs) have been performed previously [13-16]. However, when used for HDR brachytherapy source tracking, these EPID based devices are required to operate using low frame rates due to poor signal to noise ratios, and when compounded with their inherently slow read out electronics, loss of data for short dwell times can occur [14]. Furthermore, none of these studies have applied source position identification to TRUS based HDR pBT, where the presence of the TRUS probe is expected to 
attenuate the signal collected by the device significantly, negatively affecting the source tracking accuracy.

The aim of this study is to determine the feasibility of using a 2D diode array, the 'Magic Plate 900' (MP900), developed at the Centre for Medical Radiation Physics (CMRP), University of Wollongong (UoW), for in-vivo source tracking during TRUS based HDR pBT. The presence of the TRUS probe was also simulated to evaluate its effect to the source position detection. The source tracking algorithm employed in this study utilizes the relative signal of each of the diodes in the array to perform a distance calculation from the HDR source to each of the diodes in the array as input. The distance from the source to each of the diodes is used in an algorithm based on a nonlinear least squares fit method to localize the source in three dimensions. Further details of the algorithm are described in a previous study [17]. Studies performed with a previous version of the device, the 'Magic Plate 121' (MP121), in homogeneous phantom media (i.e., the presence of the TRUS probe was not modelled) have reported source localization accuracy of less than $1 \mathrm{~mm}$ [18-20] and timing resolution of 0.1 seconds [18]. A previous Monte Carlo study of the MP121, showed that source tracking accuracy with the device embedded in a carbon fibre couch below a patient in the lithotomy position was achievable to within $2 \mathrm{~mm}$ for CT based HDR pBT [17].

\section{MATERIALS AND METHODS}

To assess the feasibility of source localization using the MP900 during TRUS based HDR pBT Monte Carlo simulations were performed using the Geant4 toolkit (v4.10.01)[21, 22]. During the simulations, the Flexisource Ir-192 source (Elekta Brachytherapy, Veenendaal, The Netherlands) was simulated inside a voxelized patient geometry, and the dose deposited within the sensitive volume of each detector in the couch embedded 30x30 diode array was determined. The simulated detector dose was then used to determine the distance of all detectors in the array to each of the simulated source positions, and the array predicted source positions determined using an iterative process. The effect of 
the TRUS probe on source localization accuracy was ascertained by performing the simulations both with and without a TRUS probe present in the patient volume.

\section{II.A Ir-192 Flexisource}

The geometric model of the Flexisource used in the simulations was obtained from Granero et al.[23]. The Ir-192 core is modelled as a pure Iridium cylinder of length $3.5 \mathrm{~mm}$ and diameter $0.6 \mathrm{~mm}$, it has a physical density of $22.42 \mathrm{~g} / \mathrm{cm}^{3}$. The active Ir-192 core is surrounded by a stainless-steel shell of length $4.6 \mathrm{~mm}, 0.85 \mathrm{~mm}$ outer diameter and inner diameter of $0.67 \mathrm{~mm}$. This results in a shell thickness of $0.09 \mathrm{~mm}$. The composition by weight of the stainless-steel shell is modelled as follows: Fe 67.92\%, Cr 19\%, Ni 10\%, Mn 2\%, Si $1 \%$ and C $0.08 \%$ and the physical density is $7.999 \mathrm{~g} / \mathrm{cm}^{3}$. A more detailed description and validation of the Flexisource model used in the simulations has been presented in a previous study [17].

\section{II.BMagic Plate 900 2-D Diode Array}

The MP900 contains diodes mounted on a 0.6 mm Kapton substrate using the 'edgeless-dropin’ technique (Figure 1), as described in a study by Petasecca et al.[24]. These diodes have an improved angular dependence (less than 2\%) compared to those used in the previous design of this system, thereby simplifying the source reconstruction algorithm and improving source tracking accuracy. Furthermore, the diodes have a larger sensitive volume (higher sensitivity) to allow for enhanced signal to noise ratio at larger source-to-detector distance. The diodes are arranged in the Kapton carrier with a $7 \mathrm{~mm}$ pitch, resulting in a total array size of $21 \mathrm{~cm}$ x $21 \mathrm{~cm}$.

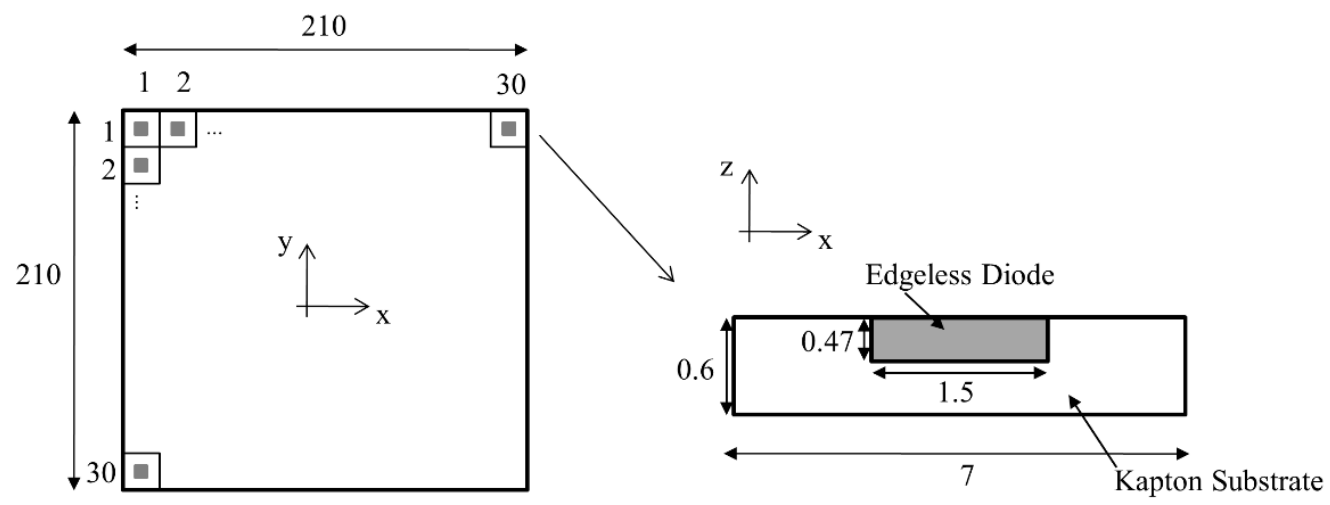


Figure 1. Schematic of the MP900 and of the 'edgeless' diode design used source tracking simulations. All dimensions are in millimetres, image is not to scale.

\section{II.C Source Tracking Simulations}

The MP900 was modelled in the source tracking simulations using the Geant4 toolkit (v4.10.01)[21, 22]. The materials and geometry of the MP900 were constructed according to the description of the device in Section II.B, embedded inside a $120 \mathrm{~mm}$ thick carbon fibre couch, offset 5 mm from its anterior surface.

The model of the patient anatomy in the simulations was created by converting DICOM CT studysets from HDR pBT treatments. This was accomplished by initially converting the Hounsfield unit (HU) to a mass density values using a CT-density curve, and then converting from mass density to a material using a look up table [25-27]. The patient geometry in Geant4 is then created as a geometrical phantom consisting of an array of voxels containing the materials (and their compositions) determined from the HU [27]. The compositions and the densities of materials used in the simulations were obtained from the AAPM TG 186 Report [28]. The voxel size was set to 3x3x3 mm in this study.

A TRUS probe was modelled inside the rectum of the voxelized patient geometries by utilizing a parallel geometry [29] to prevent overlapping volumes in the simulation, e.g. patient rectum and TRUS probe. These overlapping volumes cause errors in the tracking geometry of Geant 4 simulations, leading to erroneous results. The TRUS probe was constructed based on the Endocavity Biplane Transducer Type 8848 produced by BK Medical. The model of the probe consisted of a 110x15x3 mm bar of brass (70\% Copper, 30\% Zinc, effective atomic number of 29.5), embedded inside a cylindrical Silicon casing (length $200 \mathrm{~mm}$, diameter $22 \mathrm{~mm}$ ). It should be noted that this model may not be representative of the true construction of the model 8848 probe, as details of the internal construction of the probe were unable to be obtained from the manufacturer. Therefore, a model was used that was intended to match the internal construction of the probe to the best of our knowledge and mimicked the construction of a probe analogue previously used in experiments by our institution [30]. Due to the 
presence of the high atomic number materials inside the TRUS probe, it was expected that the TRUS probe would attenuate the signal collected by the MP900 significantly, negatively affecting the source tracking accuracy. Nevertheless, it's important to note that the TRUS probe must remain in the rectum during patient irradiation in order to not modify the patient's anatomy acquired for treatment planning [11].

Three complete HDR pBT treatments were simulated, consisting of three unique voxelized patient geometries. The source position locations from the treatment plans were re-created in the simulation geometry by using the three-dimensional source position locations obtained from the DICOM RTPlan files in the BTPS. As the patient geometry is also re-created in the simulations directly from the DICOM files, the position of the dwell locations relative to the patient anatomy in the simulations matches the BTPS. The characteristics of the three HDR pBT plans included in the study are outlined in Table I. The Flexisource model used in the simulations is described in Section II.A. The total energy deposited in the sensitive volume of each diode in the array was tallied during each simulation, which consisted of a total of $10^{9}$ primary photons. The total energy deposited in the sensitive region of each diode was then divided by the mass of the sensitive volume to calculate the dose deposited. The source positions were then determined using the average diode dose as input into an source tracking algorithm $[17,18,31]$

Table I - Characteristics of the three HDR pBT plans considered in the study.

\begin{tabular}{ccc}
\hline & Number of catheters & Number of source positions \\
\hline Patient 1 & 19 & 154 \\
Patient 2 & 22 & 165 \\
Patient 3 & 18 & 153 \\
\hline \hline
\end{tabular}

Finally, the MP900 determined source positions were compared to known source positions input into the simulations, which were obtained from the Oncentra Prostate (v4.2.3 Elekta Brachytherapy, Veenendaal, The Netherlands) BTPS. Source positions obtained from the BTPS coordinate system were converted to that of the MP900 coordinate system, as the position of the MP900 relative to the patient geometry is known, fixed distance (the MP900 is rigidly embedded into the carbon 
fibre couch below the patient) within the simulation geometry. The simulations were repeated with the TRUS probe replaced by tissue equivalent material in the patient geometry to examine the effect of the TRUS probe on the source tracking accuracy.

To determine if the same accuracy of source tracking can be achieved both with and without the TRUS probe present, a thresholding technique was applied to the source tracking algorithm. Firstly, using the data from the simulations with the TRUS probe present, the maximum dose deposited in any diode was found for each source position. Secondly, for each source position in the three patient plans a threshold was applied, ranging from $50 \%$ - 90\% of the maximum dose deposited in the array in $10 \%$ increments. Any diode with dose deposited below this threshold is ignored in the algorithm, and the optimal threshold level found that gave the best results as input into the source tracking algorithm when all source positions from the three treatment plans were considered.

The average and maximum differences between MP900 predicted and actual source positions obtained with the TRUS probe, without the TRUS probe, and TRUS probe corrected (i.e. applying the optimal threshold), were computed and compared across all three patient volumes. Results without the TRUS probe and TRUS probe corrected were also compared by means of a Wilcoxian matched pair test. A p-value of $<0.05$ was considered significant. The test was performed using the R software (v3.5.1, The R Foundation for Statistical Computing, Vienna, Austria).

\section{RESULTS}

The average three-dimensional (3D) error vector of source tracking in the presence of the TRUS probe is shown in Table II for each direction within the three patient volumes, including: the patient left-right direction (X), superior-inferior direction $(\mathrm{Y})$, and anterior-posterior direction $(\mathrm{Z})$. The average 3D error vector for all source positions in the three treatment plans was found to be $11.9 \pm 2.4 \mathrm{~mm}(\mathrm{k}=$ 1). Figure 2.a) presents the distance calculation between each diode in the MP900 array from a source position in patient 1's plan with the TRUS probe present. The colour scale represents the MP900 
calculated distance (in mm) from each diode to the source. The effect of the TRUS probe can clearly be seen in the figure, resulting in an incorrect distance calculation for the diodes shadowed by the probe.

a)

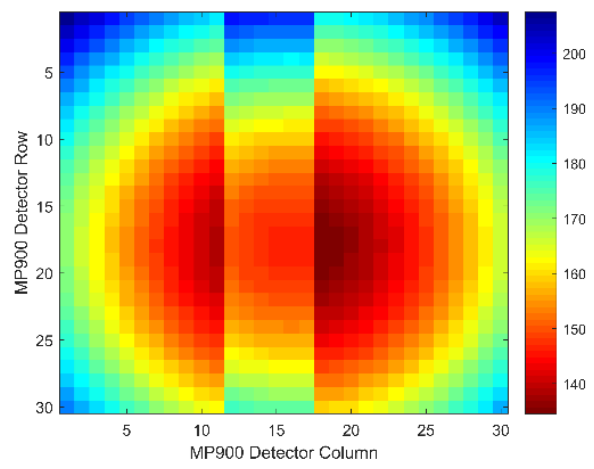

b)

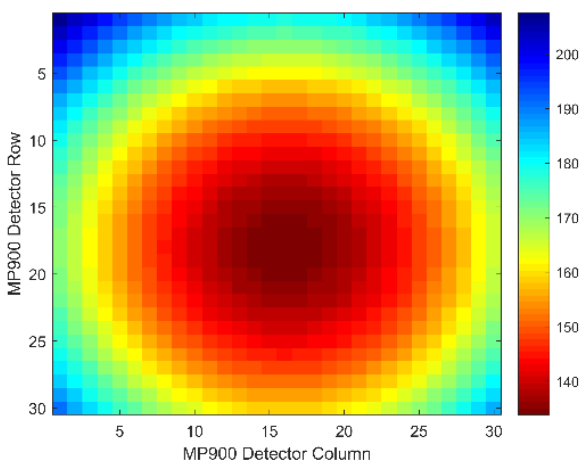

c)

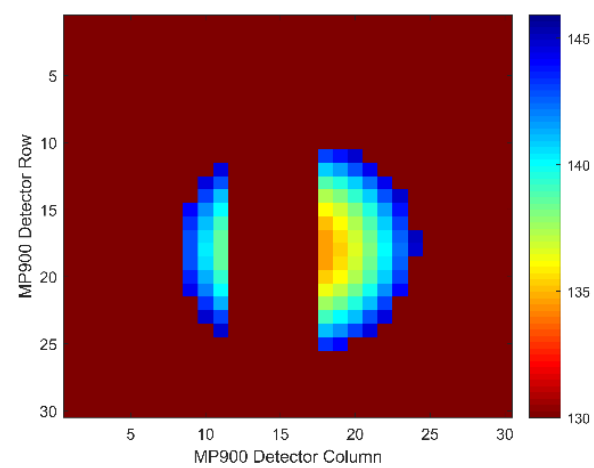

Figure 2.a) Distance calculation (mm) between the source and each diode in the MP900 from a source position in Patient 1 plan, affected by the presence of the TRUS probe.2.b) Distance calculation, without the TRUS probe present, 2.c) Distance calculation with 70\% threshold applied, and diodes with red colours not included in the reconstruction algorithm.

The simulations were then repeated without the TRUS probe present in the patient geometry examine the effect of the TRUS probe on the source tracking accuracy. Figure 2.b) presents the distance calculation between each diode in the MP900 array from a source position in patient 1's plan without the TRUS probe present. The average 3D error vector for all source positions in the three treatment plans without the TRUS probe was $1.5 \pm 0.3 \mathrm{~mm}(\mathrm{k}=1)$.

Table II - Average difference between MP900 predicted and actual source positions in mm with, without and corrected for a TRUS probe with standard deviation $(k=1)$ in the patient's rectum. 


\begin{tabular}{|c|c|c|c|c|c|c|c|c|c|}
\hline \multirow[b]{2}{*}{$\begin{array}{c}\text { Error } \\
\text { Direction }\end{array}$} & \multicolumn{3}{|c|}{ Patient 1} & \multicolumn{3}{|c|}{ Patient 2} & \multicolumn{3}{|c|}{ Patient 3} \\
\hline & $\begin{array}{l}\text { With } \\
\text { TRUS } \\
\text { Probe }\end{array}$ & $\begin{array}{c}\text { Without } \\
\text { TRUS } \\
\text { Probe }\end{array}$ & $\begin{array}{c}\text { TRUS } \\
\text { Probe } \\
\text { Corrected }\end{array}$ & $\begin{array}{l}\text { With } \\
\text { TRUS } \\
\text { Probe }\end{array}$ & $\begin{array}{c}\text { Without } \\
\text { TRUS } \\
\text { Probe }\end{array}$ & $\begin{array}{c}\text { TRUS } \\
\text { Probe } \\
\text { Corrected }\end{array}$ & $\begin{array}{c}\text { With TRUS } \\
\text { Probe }\end{array}$ & $\begin{array}{l}\text { Without } \\
\text { TRUS } \\
\text { Probe }\end{array}$ & $\begin{array}{c}\text { TRUS } \\
\text { Probe } \\
\text { Corrected }\end{array}$ \\
\hline $\bar{X}$ & $6.1 \pm 1.2$ & $0.4 \pm 0.4$ & $0.6 \pm 0.5$ & $6.6 \pm 1.4$ & $0.4 \pm 0.4$ & $0.7 \pm 0.6$ & $5.8 \pm 1.3$ & $0.5 \pm 0.4$ & $0.7 \pm 0.8$ \\
\hline $\mathrm{Y}$ & $6.0 \pm 1.4$ & $0.4 \pm 0.2$ & $0.6 \pm 0.5$ & $6.3 \pm 1.1$ & $0.4 \pm 0.2$ & $0.6 \pm 0.4$ & $6.0 \pm 1.5$ & $0.5 \pm 0.3$ & $0.7 \pm 0.7$ \\
\hline $\mathrm{Z}$ & $8.1 \pm 2.2$ & $1.3 \pm 0.2$ & $1.6 \pm 0.4$ & $7.8 \pm 2.7$ & $1.3 \pm 0.2$ & $1.4 \pm 0.4$ & $8.4 \pm 2.1$ & $1.5 \pm 0.5$ & $1.5 \pm 0.8$ \\
\hline $3 \mathrm{D}$ & $11.8 \pm 2.4$ & $1.4 \pm 0.3$ & $1.8 \pm 0.6$ & $12.0 \pm 2.5$ & $1.4 \pm 0.3$ & $1.7 \pm 0.6$ & $11.8 \pm 2.3$ & $1.7 \pm 0.4$ & $1.8 \pm 0.7$ \\
\hline
\end{tabular}

A thresholding technique was applied to the verification algorithm with the TRUS probe present. Figure 2.c) presents the distance calculation between each diode in the MP900 array from a source position in patient 1's plan with the TRUS probe present and a 70\% threshold applied. As can be seen from the figure, the threshold is able to eliminate the diodes shadowed by the TRUS probe as input into the verification algorithm. The effect of the application of different threshold levels in the source tracking algorithm is shown in Figure 3. The optimal threshold level, averaged over all source positions in the three treatment plans was found to be $70 \%$. By disregarding the signal of all diodes below $70 \%$ of the maximum signal of any diode in the array, an average source tracking accuracy of $1.8 \pm 0.4 \mathrm{~mm}$ can be achieved in the clinically relevant condition of the presence of the TRUS probe inside the rectum.

As can be seen in Table II, the variation in the 3D source tracking accuracy across the three patient volumes is less than $0.5 \mathrm{~mm}$. This result highlights the excellent reproducibility of the MP900 system and associated source tracking algorithm, regardless of variations in patient related tissue inhomogeneities. The maximum difference between MP900 predicted and actual source position was $15.4 \mathrm{~mm}, 2.2 \mathrm{~mm}$, and $3.0 \mathrm{~mm}$, with the TRUS probe, without the TRUS probe, and TRUS probe corrected (70\% threshold), respectively across all three patient volumes. The difference between the latter two datasets was found to be statistically significant, with $p<0.001$. 


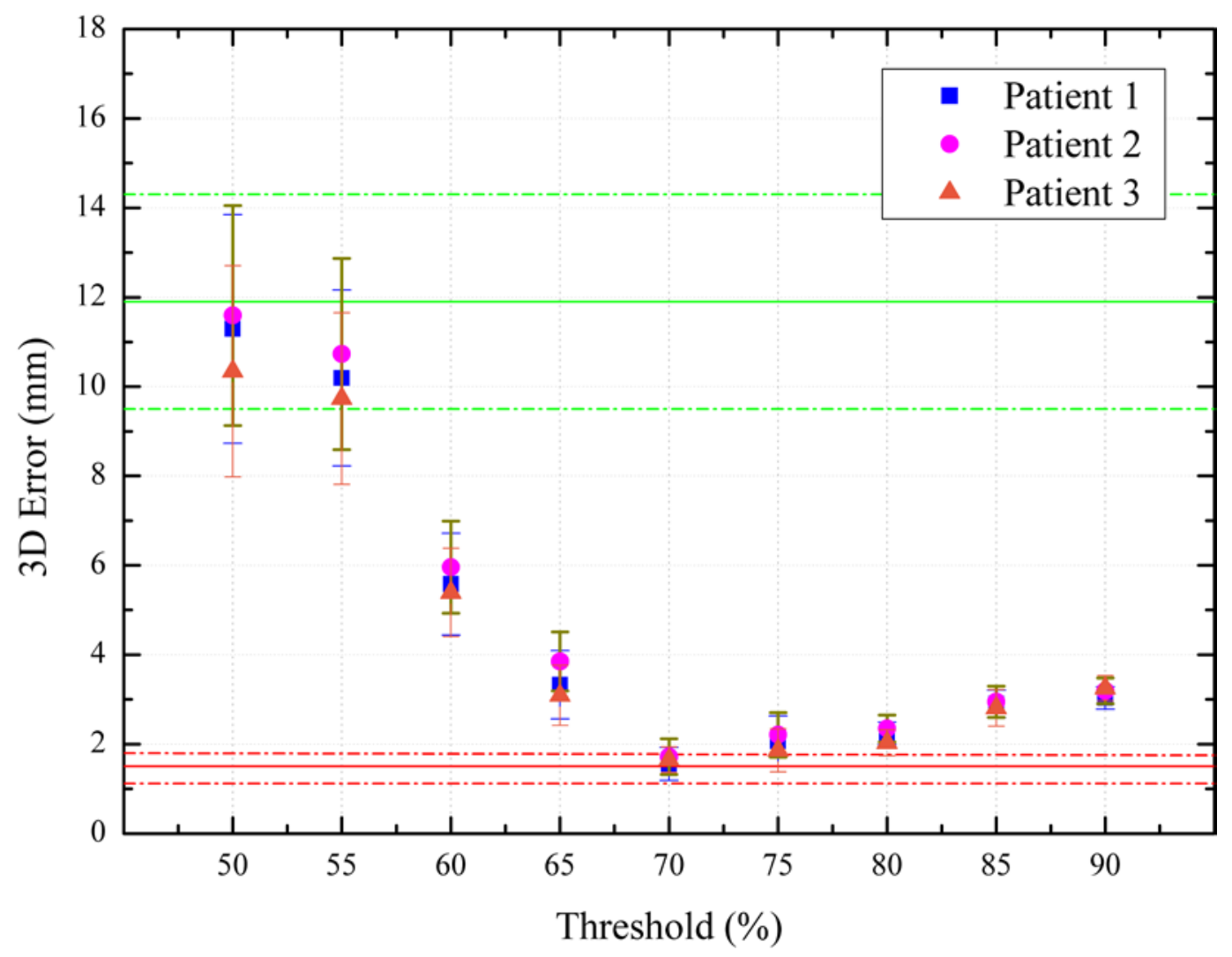

Figure 3. Effect of threshold level in the source tracking algorithm. The 3D error vector in the presence of the TRUS probe is shown in green $(\mathrm{k}=1)$. The $3 \mathrm{D}$ error vector without the TRUS probe is shown in red $(\mathrm{k}=1)$. The 3D error vector with different threshold levels applied is shown in blue, pink, and orange, for patients 1,2 and 3 , respectively $(\mathrm{k}=1)$. 


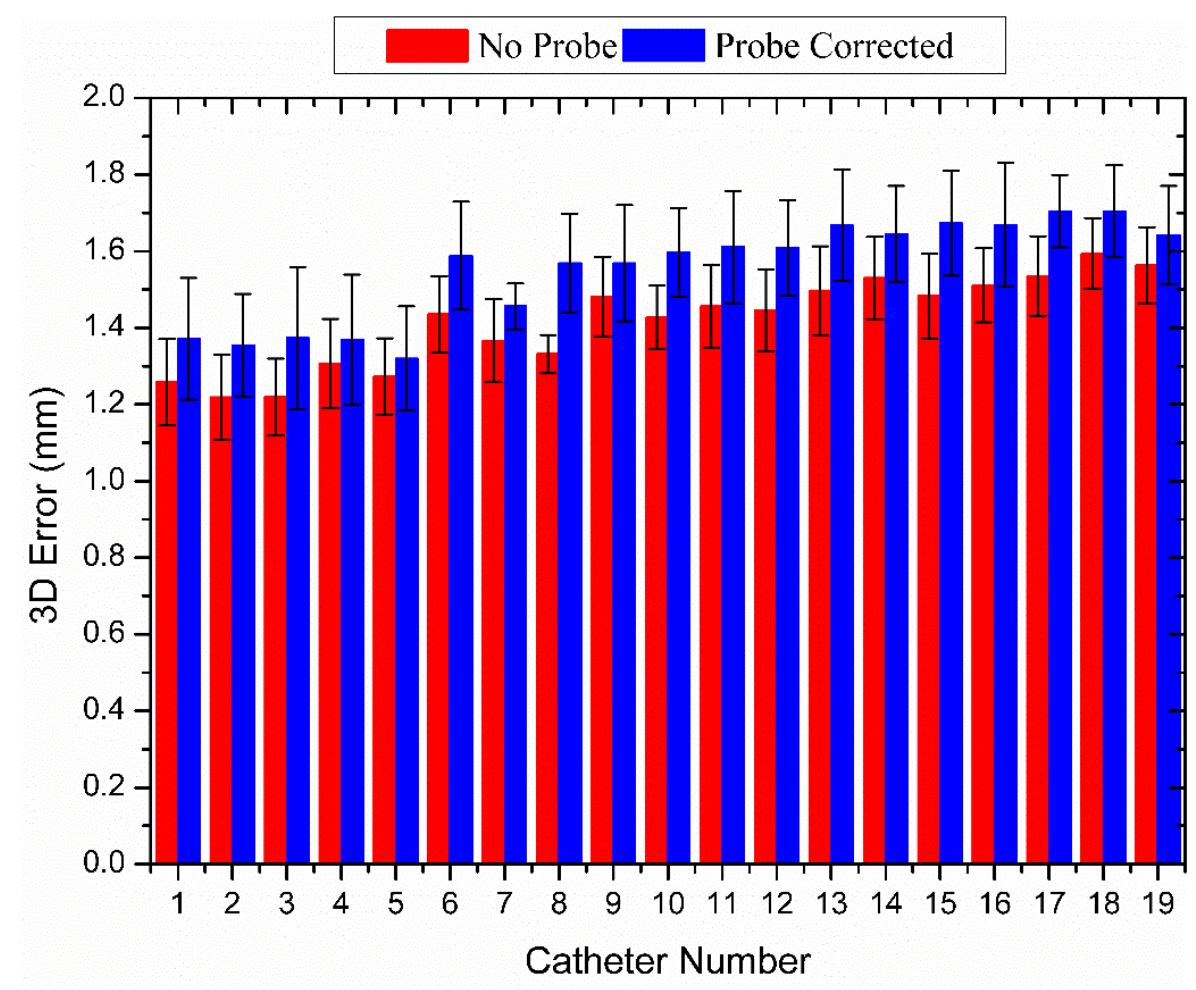

Figure 4. Average 3D source tracking error in each catheter considered in the study for patient $1, \pm 1$ standard deviation. Red columns show the average error without the TRUS probe. Blue columns show the average error with the TRUS probe present and a 70\% threshold level applied.

The results were broken down further to show the average 3D error achieved per catheter (Figure 4) for patient 1 only, both without the TRUS probe and with the 70\% threshold level applied. The catheter numbers increase in the posterior to anterior direction; therefore, the increasing catheter number represents an increasing distance between the source and the MP900 array. Typical average distances between the source positions in each catheter and the MP900 array ranged from 95 mm to 145 $\mathrm{mm}$. A trend is observed showing an increased 3D error with increasing average distance between the source and the array. 


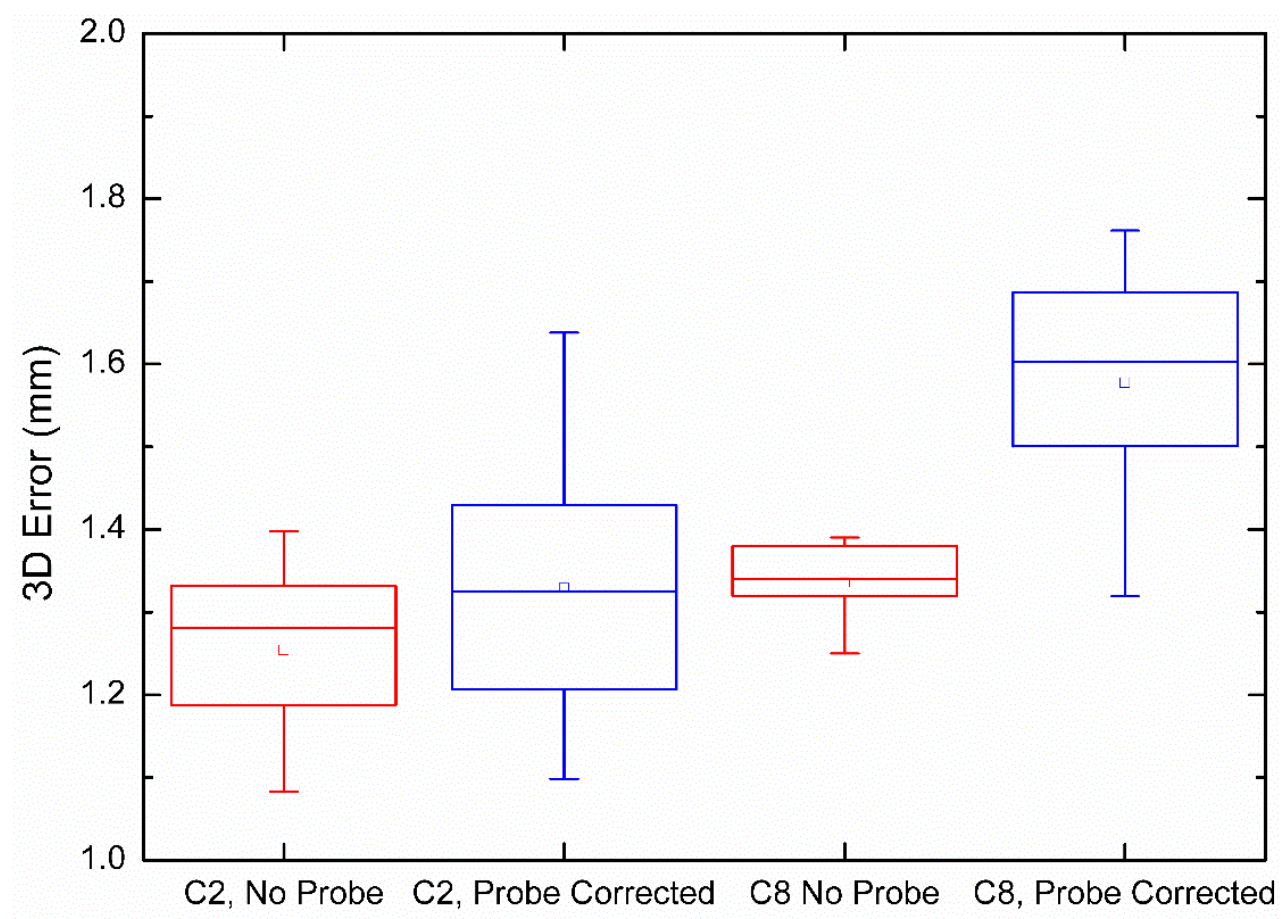

Figure 5. 3D source tracking error in the form of a box-whisker plot for catheter 2 (C2) and catheter 8 (C8). TRUS probe present and TRUS probe corrected results are presented for each catheter.

Figure 5 presents a box-whisker plot focusing on catheter 2 and catheter 8 from the patient 1 plan. Catheter 2 is an example of a catheter which is not shadowed by the TRUS probe as viewed from the MP900 array. Catheter 8 is an example where the TRUS probe shadows the primary radiation from the Ir-192 source from most detectors in the MP900 array.

\section{DISCUSSION}

The presence of the TRUS probe was found to significantly affect the accuracy of source tracking in all three dimensions. Source tracking accuracy without the TRUS probe using the MP900 was found to be similar, but improved by approximately $0.5 \mathrm{~mm}$, to that found previously using the MP121 device [17]. The major discrepancy remains in the $\mathrm{z}$ direction (patient anterior-posterior direction). The discrepancy in the $\mathrm{z}$ direction has been shown previously to be in particular due to the non-water equivalence of patient tissue related inhomogeneities between the source and the diode array. This was shown by repeating the simulations with each voxel in the geometry assigned a density of 
water, resulting in a significant improvement in source tracking accuracy [17]. This effect is highlighted even more so in Figure 4, where an increased 3D error vector is observed with increasing distance between source position and the array, due to the increasing likelihood of the material composition between the source and the array being different to that of water.

The effect of the TRUS probe on source tracking accuracy can be overcome using a thresholding technique. An optimal threshold level of $70 \%$ of the maximum diode signal was found for all source positions in three complete HDR pBT treatment plans. With this threshold of $70 \%$, approximately $60 \%$ of the detectors in the MP900 are not being used for source position verification for the reported example case (patient 1). However, the total number of detectors used in the verification algorithm under these conditions is on average equal to 315 . This is approximately 2.5 times the total number of detectors in the previous version of the device (MP121 array) and justifies the need for the MP900 for this purpose. As shown in Figure 3, the 70\% threshold level was found to be optimal for all three patient geometries, and there was remarkably little variation in source tracking accuracy between the three patient geometries. The optimal threshold level may depend more on the TRUS probe design and composition, rather than the patient geometry. The effect of the TRUS probe type and design on the optimal threshold level will be investigated further in future experimental studies with the MP900.

Source position detection is in principle impossible, even applying a threshold technique, if the source is so close to the probe that its primary radiation oriented towards the array is shielded by the probe. Defining $a$ the distance between the centre of the TRUS probe and the array, $b$ the distance between the upper surface of the TRUS probe and the source, $h$ the length of the array (i.e. $100 \mathrm{~mm}$ for the MP121 and $210 \mathrm{~mm}$ for the MP900) and $d$ the diameter of the probe (i.e. $22 \mathrm{~mm}$ for the Endocavity Biplane Transducer Type 8848 produced by BK Medical) (Figure 6.a)), the following equation $\frac{a+b+d / 2}{h}=\frac{b+d / 2}{d}$ is valid when the "shadow" of the source matches the lateral edges of the array. In Figure 6.b), this equation was used to simulate $b$ depending on both $a$ and the diode array dimensions. According to the plot, the use of MP900 allows the reconstruction of the source position even if it is over the TRUS probe and close to it, covering all the possible clinical situations. This wouldn't be 
possible with the MP121, in particular at increasing distances between TRUS probe and detector array.

A typical clinical example is shown in Figure 6.c), with a needle $10.4 \mathrm{~mm}$ over the TRUS surface.

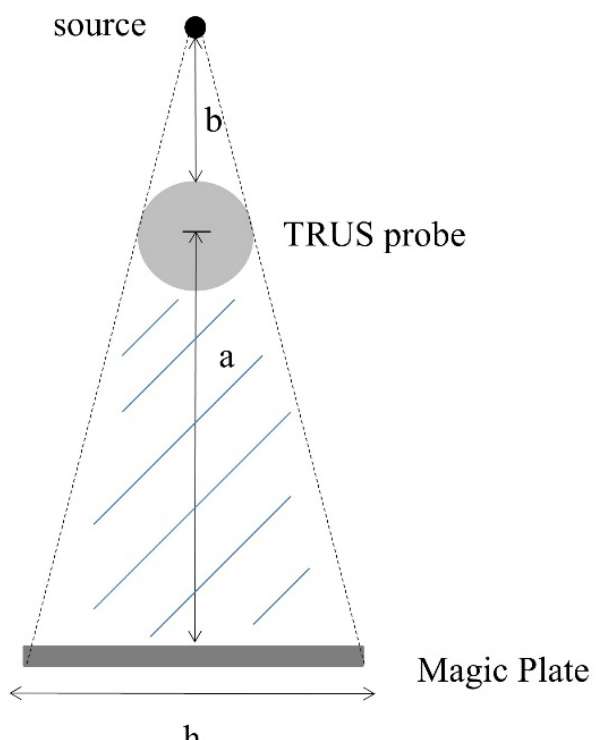

a)

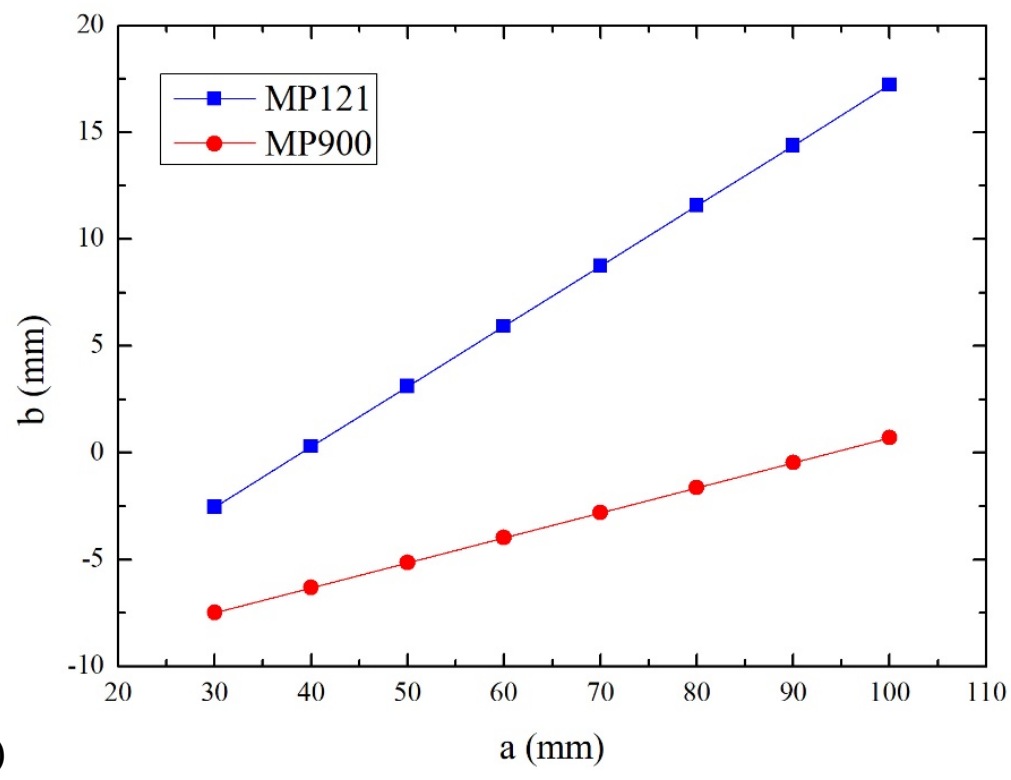




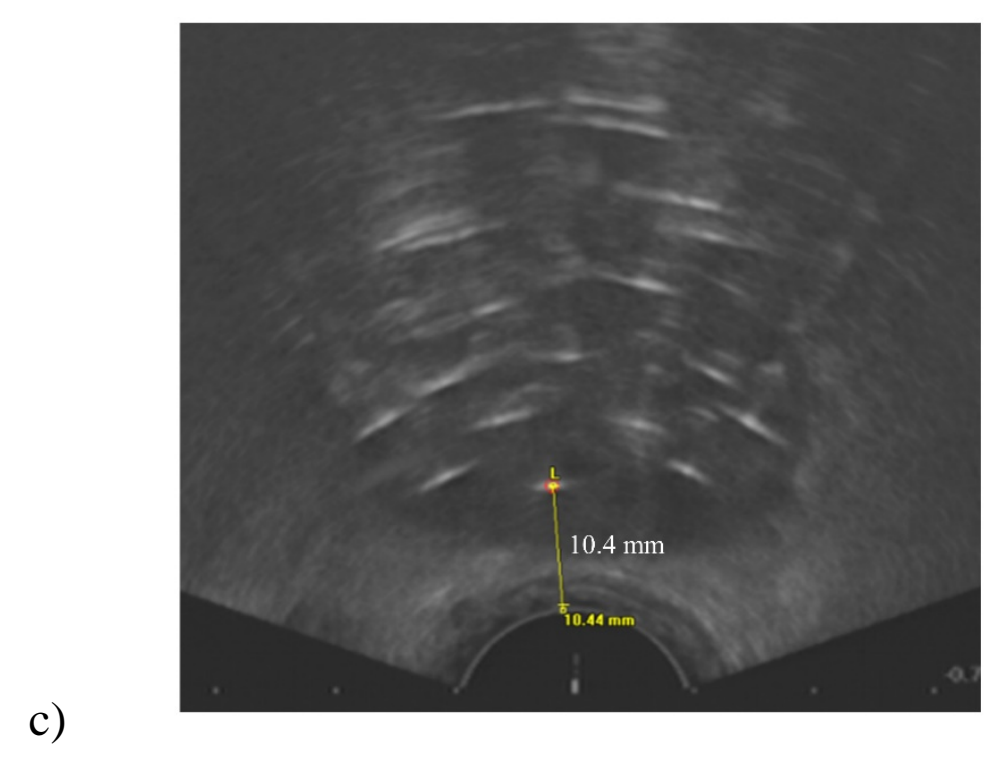

Figure 6.a) Schematic of the setup with the source, TRUS probe and Magic Plate array. $a$ is the distance between the centre of the TRUS probe and the array; $b$ is the distance between the external surface of the TRUS probe and the source; $h$ is the length of the array; $d$ is the diameter of the probe. Image is not to scale; b) Minimum source-probe distance $b$ necessary to avoid the complete shielding of the primary radiation of the source that would be detected by the array, plotted at changing array-probe distance $a$; c) TRUS image of the prostate after needle implantation. The closest needle is $10.4 \mathrm{~mm}$ over the TRUS probe.

Figure 5 shows on patient 1 an example of a catheter (catheter 2) which is not shadowed by the TRUS probe as viewed from the MP900 array and of a catheter (catheter 8) where the TRUS probe shadows the primary radiation from the Ir-192 source from most detectors in the MP900 array. Even for catheter 8 , which is positioned directly anterior to the TRUS probe, the modified source tracking algorithm results in a similar source tracking accuracy to when no probe is present. However, when compared to catheter 2, the modified source tracking algorithm applied to catheter 8 results in larger discrepancies relative to when there is no probe present. As shown in table 2, there is a higher standard deviation of the 3D error results in the TRUS probe corrected group $(0.6 \mathrm{~mm})$ compared to the results without the TRUS probe $(0.3 \mathrm{~mm})$. This is due to the larger uncertainty in correcting for the presence of the TRUS probe for catheters that are located directly above the probe where less detectors in the 
MP900 array can be used for input into the verification algorithm. In general, 3D errors resulted significantly higher $(\mathrm{p}<0.001)$ in the TRUS probe corrected group than in the group without TRUS probe. Nevertheless, an average source tracking accuracy lower than $2 \mathrm{~mm}$ (i.e., $1.8 \pm 0.4 \mathrm{~mm}$ ) can be achieved in the clinically relevant condition of the presence of the TRUS probe inside the rectum, after application of the TRUS probe correction method.

The results from this study indicate that the MP900 will have sufficient sensitivity to detect errors of approximately $2 \mathrm{~mm}$ in the delivery of TRUS based HDR pBT treatments. Such errors may include incorrect catheter connection, incorrect source strength, or incorrect reference length entered into the BTPS. Errors of this magnitude may also be due to uncertainties in reconstruction of catheters on ultrasound images, for example Rylander et al.[10] have shown that errors in catheter reconstruction on TRUS images relative to MRI may be up to $9.6 \mathrm{~mm}$. Furthermore, possible needle displacements occurring during the treatment planning phase (i.e., post-imaging intra-fraction modifications), which might result in changes in the dosimetry of the prostate and OARs [11, 32], could also be detected by the use of MP900.

There still remains, however, a number of significant challenges that must be overcome before translating these source tracking simulation studies to routine in-vivo source tracking in clinical practice. One example is the poor signal to noise ratio experienced by the detector system at such large source to detector distances, when the detector system is placed beneath the patient during HDR pBT. This poor signal to noise ratio necessitates the use of longer integration times in the detector system and may therefore preclude the system from performing real-time source tracking analysis. This issue has been discussed in previous publications on the use of EPID devices for source tracking [13-16]. The Magic Plate system, however, is capable of operating with variable integration times between $14-9900 \mu \mathrm{s}$, with a stable sampling frequency between $0.1-10 \mathrm{kHz}$. Previous publications on the application of the Magic Plate system to HDR brachytherapy source tracking have demonstrated that real-time analysis is possible $[18,19]$. Optimisation of detector integration times and sampling frequencies when performing source tracking in-vivo will be a focus of future publications. 
Another challenge that is still to be overcome is the registration of the MP900 to the BTPS coordinate system. The accuracy of source tracking can only be as good as the registration between these coordinate systems, and previous publications have incorporated stereoscopic imaging [13, 14], or electromagnetic tracking [33] in attempts to perform this registration. However, this issue remains one of the most significant challenges for accurate localisation of the source position and will also be a focus of future publications on experimental results using the MP900 system in combination with gel phantoms.

\section{CONCLUSION}

This study presents a novel methodology for performing source tracking during real-time TRUS based HDR pBT. Inclusion of the TRUS probe inside the patient was shown to negatively affect source tracking accuracy. However, modification of the source tracking algorithm using thresholding techniques was shown to improve source tracking in the presence of the TRUS probe. An optimal threshold level of $70 \%$ of the maximum diode signal at each source position was determined for all source positions in three complete HDR pBT treatment plans, with minor variation observed across the three patient volumes. Using this thresholding technique, similar accuracy can be achieved as to when the TRUS probe is not present inside the patient volume, highlighting the robustness of the proposed solution.

\section{ACKNOWLEDGEMENTS}

The authors would like to acknowledge the University of Wollongong Information Management \& Technology Services (IMTS) for computing time on the UOW High Performance Computing Cluster. This research was supported by the National Computer Merit Allocation Scheme (NCMAS) project zi60, as well as the Australian Government Research Training Program (RTP). 


\section{REFERENCES}

[1] Hoskin P, Rojas A, Ostler P, Hughes R, Lowe G, Bryant L. Quality of life after radical radiotherapy for prostate cancer: longitudinal study from a randomised trial of external beam radiotherapy alone or in combination with high dose rate brachytherapy. Clinical Oncology. 2013;25:321-7.

[2] Hoskin PJ, Motohashi K, Bownes P, Bryant L, Ostler P. High dose rate brachytherapy in combination with external beam radiotherapy in the radical treatment of prostate cancer: initial results of a randomised phase three trial. Radiotherapy and Oncology. 2007;84:114-20.

[3] Hoskin PJ, Rojas AM, Bownes PJ, Lowe GJ, Ostler PJ, Bryant L. Randomised trial of external beam radiotherapy alone or combined with high-dose-rate brachytherapy boost for localised prostate cancer. Radiotherapy and Oncology. 2012;103:217-22.

[4] Jawad MS, Dilworth JT, Gustafson GS, Ye H, Wallace M, Martinez A, et al. Outcomes Associated With 3 Treatment Schedules of High-Dose-Rate Brachytherapy Monotherapy for Favorable-Risk Prostate Cancer. International Journal of Radiation Oncology • Biology• Physics. 2016;94:657-66.

[5] Barkati M, Williams SG, Foroudi F, Tai KH, Chander S, van Dyk S, et al. High-Dose-Rate Brachytherapy as a Monotherapy for Favorable-Risk Prostate Cancer: A Phase II Trial. International Journal of Radiation Oncology • Biology • Physics. 2012;82:1889-96.

[6] Whitaker M, Hruby G, Lovett A, Patanjali N. Prostate HDR brachytherapy catheter displacement between planning and treatment delivery. Radiotherapy and Oncology. 2011;101:490-4.

[7] Poder J, Whitaker M. Robustness of IPSA optimized high-dose-rate prostate brachytherapy treatment plans to catheter displacements. Journal of contemporary brachytherapy. 2016;8:201.

[8] Doyle AJ, King DM, Browne JE. A review of the recommendations governing quality assurance of ultrasound systems used for guidance in prostate brachytherapy. Physica Medica. 2017;44:51-7.

[9] Batchelar DL, Chung HT, Loblaw A, Law N, Cisecki T, Morton GC. Intraoperative ultrasoundbased planning can effectively replace postoperative CT-based planning for high-dose-rate brachytherapy for prostate cancer. Brachytherapy. 2016;15:399-405. 
[10] Rylander S, Buus S, Pedersen EM, Bentzen L, Tanderup K. Dosimetric impact of contouring and needle reconstruction uncertainties in US-, CT- and MRI-based high-dose-rate prostate brachytherapy treatment planning. Radiotherapy and Oncology. 2017;123:125-32.

[11] Carrara M, Tenconi C, Rossi G, Borroni M, Cerrotta A, Grisotto S, et al. In vivo rectal wall measurements during HDR prostate brachytherapy with MOSkin dosimeters integrated on a trans-rectal US probe: Comparison with planned and reconstructed doses. Radiotherapy and Oncology. 2016;118:148-53.

[12] Mason J, Mamo A, Al-Qaisieh B, Henry AM, Bownes P. Real-time in vivo dosimetry in high dose rate prostate brachytherapy. Radiotherapy and Oncology. 2016;120:333-8.

[13] Smith RL, Haworth A, Panettieri V, Millar JL, Franich RD. A method for verification of treatment delivery in HDR prostate brachytherapy using a flat panel detector for both imaging and source tracking. Medical physics. 2016;43:2435-42.

[14] Smith RL, Hanlon M, Panettieri V, Millar JL, Matheson B, Haworth A, et al. An integrated system for clinical treatment verification of HDR prostate brachytherapy combining source tracking with pretreatment imaging. Brachytherapy. 2018;17:111-21.

[15] Smith RL, Haworth A, Panettieri V, Millar JL, Franich RD. 3D catheter reconstruction in HDR prostate brachytherapy for pre-treatment verification using a flat panel detector. Physica Medica. 2017;39:121-31.

[16] Fonseca GP, Podesta M, Bellezzo M, Van den Bosch MR, Lutgens L, Vanneste BG, et al. Online pretreatment verification of high-dose rate brachytherapy using an imaging panel. Physics in Medicine \& Biology. 2017;62:5440.

[17] Poder J, Cutajar D, Guatelli S, Petasecca M, Howie A, Bucci J, et al. HDR brachytherapy in vivo source position verification using a 2D diode array: A Monte Carlo study. Journal of applied clinical medical physics. 2018;19:163-72.

[18] Espinoza A, Petasecca M, Fuduli I, Howie A, Bucci J, Corde S, et al. The evaluation of a 2D diode array in "magic phantom" for use in high dose rate brachytherapy pretreatment quality assurance. Medical Physics. 2015;42:663-73. 
[19] Espinoza A, Petasecca M, Cutajar D, Fuduli I, Howie A, Bucci J, et al. Pretreatment verification of high dose rate brachytherapy plans using the 'magic phantom' system. Biomedical Physics \& Engineering Express. 2015;1:025201.

[20] Espinoza A, Beeksma B, Petasecca M, Fuduli I, Porumb C, Cutajar D, et al. The feasibility study and characterization of a two-dimensional diode array in "magic phantom" for high dose rate brachytherapy quality assurance. Medical Physics. 2013;40:111702.

[21] Agostinelli S, Allison J, Amako K, Apostolakis J, Araujo H, Arce P, et al. Geant4—a simulation toolkit. Nuclear Instruments and Methods in Physics Research Section A: Accelerators, Spectrometers, Detectors and Associated Equipment. 2003;506:250-303.

[22] Allison J, Amako K, Apostolakis J, Araujo H, Dubois PA, Asai M, et al. Geant4 developments and applications. IEEE Transactions on Nuclear Science. 2006;53:270-8.

[23] Granero D, Pérez-Calatayud J, Casal E, Ballester F, Venselaar J. A dosimetric study on the Ir-192 high dose rate Flexisource. Medical Physics. 2006;33:4578-82.

[24] Petasecca M, Alhujaili S, Aldosari A, Fuduli I, Newall M, Porumb C, et al. Angular independent silicon detector for dosimetry in external beam radiotherapy. Medical physics. 2015;42:4708-18.

[25] Kimura A, Aso T, Yoshida H, Kanematsu N, Tanaka S, Sasaki T. DICOM data handling for Geant4based medical physics application. Nuclear Science Symposium Conference Record, 2004 IEEE: IEEE; 2004. p. 2124-7.

[26] Aso T, Kimura A, Yamashita T, Sasaki T. Optimization of patient geometry based on ct data in geant4 for medical application. 2007 IEEE Nuclear Science Symposium Conference Record: IEEE; 2007. p. 2576-80.

[27] Giacometti V, Guatelli S, Bazalova-Carter M, Rosenfeld AB, Schulte RW. Development of a high resolution voxelised head phantom for medical physics applications. Physica Medica. 2017;33:182-8. [28] Beaulieu L, Carlsson Tedgren Å, Carrier JF, Davis SD, Mourtada F, Rivard MJ, et al. Report of the Task Group 186 on model-based dose calculation methods in brachytherapy beyond the TG-43 formalism: Current status and recommendations for clinical implementation. Medical physics. 2012;39:6208-36. 
[29] Apostolakis J, Asai M, Cosmo G, Howard A, Ivanchenko V, Verderi M. Parallel Geometries in Geant4: foundation and recent enhancements. Proceedings of the 2008 IEEE NSS/MIC/RTSD Conference2008.

[30] Espinoza AA. The development of a silicon detector quality assurance system for high dose rate brachytherapy: University of Wollongong; 2016.

[31] Rozenfeld A, Zaider M. Method and apparatus for real time dosimetry. EP1481265B1. Google Patents; 2008.

[32] Milickovic N, Mavroidis P, Tselis N, Nikolova I, Katsilieri Z, Kefala V, et al. 4D analysis of influence of patient movement and anatomy alteration on the quality of 3D U/S-based prostate HDR brachytherapy treatment delivery. Medical physics. 2011;38:4982-93.

[33] Beaulieu L, Racine E, Han DY, Vigneault E, Hsu I-C, Cunha JAM. Real-time electromagnetic tracking-based treatment platform for high-dose-rate prostate brachytherapy: Clinical workflows and end-to-end validation. Brachytherapy. 2017. 